Research, Society and Development, v. 10, n. 3, e4110312934, 2021

(CC BY 4.0) | ISSN 2525-3409 | DOI: http://dx.doi.org/10.33448/rsd-v10i3.12934

\title{
Avaliação do perfil epidemiológico das pacientes com Doença Trofoblástica \\ Gestacional atendidas no Centro de Referência de Sergipe
}

\author{
Evaluation of the epidemiological profile of patients with Gestational Trophoblastic Disease treated \\ at the Reference Center of Sergipe \\ Evaluación del perfil epidemiológico de pacientes con Enfermedad Trofoblástica Gestacional \\ atendidas en el Centro de Referencia de Sergipe
}

Recebido: 13/02/2021 | Revisado: 21/02/2021 | Aceito: 26/02/2021 | Publicado: 04/03/2021

\author{
Mariana Moscoso Rêgo de Matos \\ ORCID: https://orcid.org/0000-0003-2032-7900 \\ Universidade Tiradentes, Brasil \\ E-mail: mariana.moscoso@souunit.com.br \\ Marina de Pádua Nogueira Menezes \\ ORCID: https://orcid.org/0000-0002-3936-7470 \\ Universidade Tiradentes, Brasil \\ E-mail: marinapnogueira@yahoo.com.br \\ Larissa Maria Cardoso Lima Rodrigues \\ ORCID: https://orcid.org/0000-0003-2781-2298 \\ Universidade Federal de Sergipe, Brasil \\ E-mail: larissa cardoso97@yahoo.com.br \\ Maria Nathália Prado Simões Mendonça \\ ORCID: https://orcid.org/0000-0001-8529-7883 \\ Universidade Tiradentes, Brasil \\ E-mail: nathaliaprado23@gmail.com
}

\begin{abstract}
Resumo
Objetivo: Descrever o perfil epidemiológico das pacientes com Doença Trofoblástica Gestacional (DTG) em Sergipe. Métodos: Trata-se de um estudo observacional, descritivo, colaborativo, realizado com pacientes do sexo feminino com diagnóstico de DTG através da aplicação de questionário. Foram avaliadas 86 mulheres com DTG atendidas no centro de referência em Sergipe, no Hospital Universitário da Universidade Federal de Sergipe (HU-UFS). Resultados: Após análise dos 86 questionários incluídos, foi observado que maioria das pacientes estudadas são mulheres com idade igual ou superior a 35 anos $(57,1 \%)$, a cor parda foi a de maior prevalência $(77,8 \%)$ e em grande parte católicas $(52,7 \%)$. Quanto ao nível de escolaridade até o $2^{\circ}$ grau completo foi o mais comum $(37,2 \%)$ mais da metade já haviam apresentado uma gestação prévia $(60,5 \%)$. Em sua grande maioria possuem uma parceria $(92,5 \%)$ renda mensal familiar de até 1 salário mínimo $(73,6 \%)$ e residentes do interior do estado de Sergipe $(70,0 \%)$. Conclusão: O perfil traçado foi de mulheres com idade igual ou superior a 35 anos, pardas, católicas, com escolaridade até o $2^{\circ}$ grau completo, com história prévia de gestação, com parceria presente, com renda mensal até 1 salário mínimo e que veem do interior de Sergipe para receber tratamento no Centro de Referência do Estado para DTG.
\end{abstract}

Palavras-chave: Doença trofoblástica gestacional; Epidemiologia; Perfil de saúde.

\begin{abstract}
Objective: To describe the epidemiological profile of patients with Gestational Trophoblastic Disease (DTG) in Sergipe. Methods: This is an observational, descriptive, collaborative study, carried out with female patients diagnosed with GDD through the application of a questionnaire. 86 women with GDD treated at the referral center in Sergipe, at the University Hospital of the Federal University of Sergipe (HU-UFS), were evaluated. Results: After analyzing the 86 questionnaires included, it was observed that most of the studied patients are women aged 35 years or over (57.1\%), brown skin was the most prevalent (77.8\%) and in great Catholic (52.7\%). As for the level of education up to high school, it was the most common (37.2\%), more than half of whom had already had a previous pregnancy (60.5\%). Most of them have a partnership (92.5\%) monthly family income of up to 1 minimum wage (73.6\%) and residents of the interior of the state of Sergipe (70.0\%). Conclusion: The profile drawn was women aged 35 years or older, mixed race, Catholic, with schooling up to complete high school, with a previous pregnancy history, with a present partnership, with monthly income up to 1 minimum wage and who come from interior of Sergipe to receive treatment at the State Reference Center for DTG.
\end{abstract}

Keywords: Gestational trophoblastic disease; Epidemiology; Health profile. 


\begin{abstract}
Resumen
Objetivo: Describir el perfil epidemiológico de pacientes con Enfermedad Trofoblástica Gestacional (DTG) en Sergipe. Métodos: Se trata de un estudio observacional, descriptivo, colaborativo, realizado con pacientes del sexo femenino diagnosticadas de GDD mediante la aplicación de un cuestionario. Se evaluaron 86 mujeres con GDD atendidas en el centro de referencia de Sergipe, en el Hospital Universitario de la Universidad Federal de Sergipe (HU-UFS). Resultados: Tras analizar los 86 cuestionarios incluidos, se observó que la mayoría de los pacientes estudiados son mujeres de 35 años o más $(57,1 \%)$, la piel morena fue la más prevalente $(77,8 \%)$ y en gran medida Católico $(52,7 \%)$. En cuanto al nivel de estudios hasta el bachillerato, fue el más común $(37,2 \%)$, más de la mitad de los cuales ya había tenido un embarazo anterior $(60,5 \%)$. La mayoría de ellos tienen un ingreso familiar mensual asociado $(92,5 \%)$ de hasta 1 salario mínimo $(73,6 \%)$ y residentes del interior del estado de Sergipe (70,0\%). Conclusión: El perfil elaborado fue el de mujeres de 35 años o más, mestizas, católicas, con escolaridad hasta el bachillerato completo, con antecedente de embarazo previo, con pareja actual, con ingresos mensuales hasta 1 salario mínimo y que provienen de interior de Sergipe para recibir tratamiento en el Centro Estatal de Referencia para DTG.

Palabras clave: Enfermedad trofoblástica gestacional; Epidemiología; Perfil de salud.
\end{abstract}

\title{
1. Introdução
}

A Doença Trofoblástica Gestacional (DTG) pode ser definida como uma anomalia proliferativa que acomete as células que compõem o tecido trofoblástico placentário (Braga, et al., 2018). Ela inclui a Mola Hidatiforme Completa ou Incompleta e as apresentações malignas como a Mola Invasora, o Coriocarcinoma e o Tumor Trofoblástico do sítio Placentário. Apesar do processo etiológico permanecer desconhecido, acredita-se que a sua origem seja por uma anormalidade no processo de gametogênese e fertilização, podendo ser associada a transformação maligna do tecido trofoblástico (Ngan, et al., 2018)

A Organização Mundial da Saúde (OMS) classifica a doença trofoblástica gestacional em diferentes entidades histopatológicas, são elas: Mola Hidatiforme (Mola Completa ou Incompleta), Neoplasia Trofoblástica Gestacional (Mola Invasora, Coriocarcinoma Gestacional, Tumor Trofoblástico do Sítio Placentário, Tumor Trofoblástico Epitelioide) e Outras Lesões Trofoblásticas (Nódulo de Sítio Placentário, Sítio Placentário Exagerado).

É caracterizada por sangramento vaginal irregular, associado ou não ao atraso menstrual, com um nível extremamente alto da porção beta da gonadotrofina coriônica humana ( $\beta$-hCG) (Sun, et al., 2016). Na Mola Hidatiforme, na Mola Invasora e no Coriocarcinoma, há uma correlação direta entre os valores de $\beta$-hCG, o número de células trofoblásticas e a carga tumoral (Zugaib, 2020). Dessa forma, o $\beta$-hCG é utilizado como um dos parâmetros para diagnóstico e acompanhamento da DTG. A hiperêmese gravídica, comum nos casos de mola volumosa, também parece estar relacionada aos altos títulos de hCG encontrados nessa doença. (Khoo, et al., 2003).

Sua prevalência apresenta variações em diferentes regiões do mundo. Além disso, mudanças na epidemiologia da DTG já foram observadas em vários países (Ngan, et al., 2018). A Mola Hidatiforme (HM), forma mais comum da DTG, tem uma frequência de 1 a cada 1.000 gestações na América do Norte e Europa e acredita-se que no Brasil a incidência seja de 2 a 3 vezes maior. (Braga, et al., 2019).

Sabe-se que os extremos da idade reprodutiva e o histórico de gravidez molar anterior são importantes fatores de risco para DTG (Ngan, et al., 2018). A adolescência e a idade materna avançada foram associadas ao risco aumentado de mola completa, mas não de mola parcial (Gockley, et al., 2016). Além disso, um estudo transversal feito com 255 gestações molares e mais de 105.000 nascidos vivos demonstrou que etnia também é um significante fator de risco para gravidez molar completa e parcial (Melamed, et al., 2016)

Ireson, et al. (2018) realizou uma revisão sistemática da qualidade de vida das pacientes em algum momento diagnosticadas com DTG e os resultados sugerem a presença de sequelas físicas, psicológicas e sociais importantes associadas, com níveis clinicamente significativos de ansiedade, depressão, disfunção sexual e preocupações com a fertilidade. Portanto, mesmo que a DTG não seja uma doença tão comum entre as mulheres, quando ela está presente seu impacto na saúde e na qualidade de vida é de grande importância. Desse modo, faz-se necessário que saibamos identificar de forma precoce, tratar e 
dar todo suporte necessário a essas pacientes.

Logo, esse estudo presente será importante para ajudar a traçar o perfil epidemiológico das pacientes acometidas por DTG em Sergipe, assim como os mais prevalentes fatores de risco.

\section{Metodologia}

Foi realizado um estudo observacional, descritivo, colaborativo, quali-quantitativo (Pereira et al., 2018), com pacientes do sexo feminino com diagnóstico de DTG através da aplicação de questionário com perguntas referentes ao perfil epidemiológico da paciente como idade, etnia, religião, escolaridade, situação conjugal, renda familiar, procedência, número de gestações e paridade. Além dos dados referentes ao perfil epidemiológico das pacientes, também foi analisada a forma de esvaziamento uterino. A avaliação ocorreu após aprovação no Comitê de Ética em Pesquisa (CEP) da UFS com 86 mulheres com diagnóstico de DTG consoante aos critérios da Federação Internacional de Ginecologia e Obstetrícia (FIGO) atendidas no Centro de Referência em Sergipe para DTG, no Hospital Universitário da Universidade Federal de Sergipe (HU-UFS) de fevereiro de 2017 a setembro de 2018. Foram excluídas pacientes que engravidaram durante o seguimento.

Os dados foram compilados no programa Microsoft Office Excel. Foram obtidas variáveis qualitativas nominais e variáveis quantitativas, sendo, então, realizada uma análise descritiva dos dados. Para as variáveis qualitativas a análise descritiva procedeu com a categorização dos dados e obtenção das respectivas frequências e percentuais, e para as variáveis quantitativas foram calculadas medidas de tendência central (média), variabilidade (desvio padrão) e posição (mínimo e máximo).

O software utilizado foi o R, versão 3.6.1 (THE R CORE TEAM, 2020).

\section{Resultados}

Neste estudo foram avaliadas 86 pacientes com idade que variou entre 14 a 51 anos, com uma idade média de 26,7 $( \pm 8,7)$ anos. Destas, $26,2 \%$ apresentavam idade inferior a 20 anos, $16,7 \%$ entre 20 e 34 anos e a maior parte das mulheres, $57,1 \%$, igual ou superior a 35 anos.

No que se refere a cor/raça, 77,8\% declarou-se de cor parda, $9,7 \%$ brancas, $8,3 \%$ negras, $4,2 \%$ amarelas.

Quanto a religião, a maior parte das mulheres declararam-se católicas (52,7\%), enquanto 23,6\% de outras religiões e 23,6 \% afirmaram não possuir uma religião.

Quanto ao grau de escolaridade, $26,7 \%$ tinham até o primeiro grau completo, $37,2 \%$ até o segundo grau completo, $8,1 \%$ até o terceiro grau completo.

Sobre a situação conjugal, 92,5 \% afirmaram possuir uma parceria e 7,5\% não estavam em um relacionamento.

Quanto a renda familiar mensal, 73,6\% declararam receber até 1 salário-mínimo, 18,9\% apresentavam até 2 saláriosmínimos, 5,7\% declararam renda mensal maior que 2 salários mínimos e apenas 1,9\% não possuíam renda fixa.

Em relação à localidade de moradia, 70,0\% das pacientes do estudo pertenciam a municípios do interior de Sergipe, 25,0\% residiam na capital Aracaju e 5,0\% eram de outros estados (Bahia e Alagoas).

Em relação ao número de gestações, $39,5 \%$ estavam em sua primeira gestação e $60,5 \%$ tinha tido pelo menos uma gestação anterior àquela.

Todos esses dados podem ser observados na Tabela 1, juntamente com a média, a mediana, o desvio padrão e os valores máximos e mínimos encontrados. 
Research, Society and Development, v. 10, n. 3, e4110312934, 2021

(CC BY 4.0) | ISSN 2525-3409 | DOI: http://dx.doi.org/10.33448/rsd-v10i3.12934

Tabela 1: Perfil socioeconômico e epidemiológico das pacientes.

\begin{tabular}{|c|c|c|c|c|c|c|}
\hline Variáveis & $\mathbf{N}$ & Mínimo & Máximo & Mediana & Média & Desvio padrão \\
\hline IDADE & 84 & 14,0 & 51,0 & 26,0 & 26,7 & 8,7 \\
\hline $\mathbf{N}^{\circ}$ DE GESTAÇÕES & 86 & 1,0 & 6,0 & 2,0 & 2,2 & 1,3 \\
\hline PARIDADE & 86 & 0,0 & 4,0 & 1,0 & 1,0 & 1,1 \\
\hline Variável/ Categoria & & $\mathbf{N}$ & & & $\%$ & \\
\hline \multicolumn{7}{|l|}{ Faixa Etária } \\
\hline$<20$ anos & & 22 & & & 26,2 & \\
\hline 20 a 34 anos & & 14 & & & 16,7 & \\
\hline$\geq 35$ anos & & 48 & & & 57,1 & \\
\hline \multicolumn{7}{|l|}{ Etnia } \\
\hline Parda & & 56 & & & 77,8 & \\
\hline Branca & & 7 & & & 9,7 & \\
\hline Negra & & 6 & & & 8,3 & \\
\hline Amarela & & 3 & & & 4,2 & \\
\hline \multicolumn{7}{|l|}{ Etnia } \\
\hline Branca & & 10 & & & 13,9 & \\
\hline Não Branca & & 62 & & & 86,1 & \\
\hline \multicolumn{7}{|l|}{ Religião } \\
\hline Católica & & 29 & & & 52,7 & \\
\hline Outras religiões & & 13 & & & 23,6 & \\
\hline SEM religião & & 13 & & & 23,6 & \\
\hline \multicolumn{7}{|l|}{ Escolaridade } \\
\hline $1^{\circ}$ grau incompleto $/ 1^{\circ}$ grau completo & & 23 & & & 26,7 & \\
\hline $2^{\circ}$ grau incompleto $/ 2^{\circ}$ grau completo & & 32 & & & 37,2 & \\
\hline 3 grau incompleto $/ 3^{\circ}$ grau completo & & 7 & & & 8,1 & \\
\hline \multicolumn{7}{|l|}{ Situação conjugal } \\
\hline COM parceiro & & 62 & & & 92,5 & \\
\hline SEM parceiro & & 5 & & & 7,5 & \\
\hline \multicolumn{7}{|l|}{ Renda Familiar } \\
\hline$\leq 1$ salário mínimo & & 39 & & & 73,6 & \\
\hline 1 a 2 salários mínimos & & 10 & & & 18,9 & \\
\hline > 2 salários mínimos & & 3 & & & 5,7 & \\
\hline Sem renda fixa & & 1 & & & 1,9 & \\
\hline \multicolumn{7}{|l|}{ Cidade } \\
\hline Aracaju & & 20 & & & 25,0 & \\
\hline Outros municípios de Sergipe & & 56 & & & 70,0 & \\
\hline Outros estados & & 4 & & & 5,0 & \\
\hline \multicolumn{7}{|l|}{ Gestações } \\
\hline 1 gestação & & 34 & & & 39,5 & \\
\hline 2 ou mais & & 52 & & & 60,5 & \\
\hline \multicolumn{7}{|l|}{ Paridade } \\
\hline Nenhuma & & 37 & & & 43,0 & \\
\hline 1 paridade & & 26 & & & 30,2 & \\
\hline mais de 1 & & 23 & & & 26,7 & \\
\hline
\end{tabular}

Fonte: Autores.

Na Tabela 1 estão descritas as variáveis qualitativas-quantitativas do perfil socioeconômico e epidemiológico das pacientes estudadas: idade, faixa etária, etnia, religião, escolaridade, situação conjugal, renda familiar, cidade de procedência, número de gestações e paridade.

No que se refere a forma de esvaziamento uterino, a maioria das mulheres foram submetidas a um único método, apenas a Curetagem ou Aspiração Manual Intrauterina (AMIU), 51,9\% e 30,4\%, respectivamente.

Quando avaliado o uso da Curetagem e o AMIU, seja como único método ou combinação deles dois, verificou-se que $65,8 \%$ das mulheres utilizaram a curetagem e 43,0\% AMIU.

Sobre os tipos de diagnósticos, 38,4\% dos diagnósticos eram do tipo mola completa, 30,2\% mola incompleta, 15,1\% beta persistente, $12,8 \%$ tiveram anatomopatológico desconhecido e apenas 3,5\% neoplasias trofoblástica. Vale destacar que em 11 pacientes houve evolução do diagnóstico para neoplasia trofoblástica (Tabela 2). 
Research, Society and Development, v. 10, n. 3, e4110312934, 2021

(CC BY 4.0) | ISSN 2525-3409 | DOI: http://dx.doi.org/10.33448/rsd-v10i3.12934

Tabela 2: Distribuição de frequência da forma de esvaziamento uterino e diagnóstico das pacientes.

\begin{tabular}{|c|c|c|}
\hline Variável/ Categoria & $\mathbf{N}$ & $\%$ \\
\hline \multicolumn{3}{|l|}{ FORMA DE ESVAZIAMENTO UTERINO } \\
\hline curetagem & 41 & 51,9 \\
\hline AMIU & 24 & 30,4 \\
\hline curetagem + AMIU / AMIU + curetagem & 9 & 11,4 \\
\hline AMIU + histerectomia & 1 & 1,3 \\
\hline aspiração a vácuo & 1 & 1,3 \\
\hline curetagem + histerectomia & 1 & 1,3 \\
\hline curetagem + ooforectomia e sapingectomia esquerda & 1 & 1,3 \\
\hline parto cesáreo & 1 & 1,3 \\
\hline \multicolumn{3}{|l|}{ AMIU } \\
\hline Não & 45 & 57,0 \\
\hline Sim & 34 & 43,0 \\
\hline \multicolumn{3}{|l|}{ curetagem } \\
\hline Não & 27 & 34,2 \\
\hline Sim & 52 & 65,8 \\
\hline \multicolumn{3}{|l|}{ DIAGNÓSTICO } \\
\hline mola completa & 24 & 27,9 \\
\hline mola incompleta & 23 & 26,7 \\
\hline beta persistente & 13 & 15,1 \\
\hline anatomopatológico desconhecido & 11 & 12,8 \\
\hline mola completa + evolução para neoplasia trofoblástica & 8 & 9,3 \\
\hline neoplasia trofoblástica & 3 & 3,5 \\
\hline mola incompleta + evolução para neoplasia trofoblástica & 2 & 2,3 \\
\hline mola completa (gemelar molar) & 1 & 1,2 \\
\hline mola incompleta + mola recorrente & 1 & 1,2 \\
\hline \multicolumn{3}{|l|}{ DIAGNÓSTICO_cat } \\
\hline mola completa & 33 & 38,4 \\
\hline mola incompleta & 26 & 30,2 \\
\hline beta persistente & 13 & 15,1 \\
\hline anatomopatológico desconhecido & 11 & 12,8 \\
\hline neoplasia trofoblástica & 3 & 3,5 \\
\hline \multicolumn{3}{|l|}{ Evolução para neoplasia } \\
\hline Não & 75 & 87,2 \\
\hline Sim & 11 & 12,8 \\
\hline
\end{tabular}

Fonte: Autores.

Na Tabela 2, podem ser observadas as variáveis qualitativas em números de casos e porcentagens relativas à forma de esvaziamento uterino, ao diagnóstico encontrado a partir do exame anatomopatológico e à evolução para neoplasia.

A Tabela 3 traz uma comparação entre os dados socioeconômicos e epidemiológicos com o diagnóstico a partir do anatomopatológico da paciente. Os percentuais são calculados em função da coluna. Assim, a soma dos percentuais em cada coluna será igual a $100 \%$. 
Research, Society and Development, v. 10, n. 3, e4110312934, 2021

(CC BY 4.0) | ISSN 2525-3409 | DOI: http://dx.doi.org/10.33448/rsd-v10i3.12934

Tabela 3: Distribuição de frequência do diagnóstico das pacientes em função das características socioeconômica e epidemiológica.

\begin{tabular}{|c|c|c|c|c|c|}
\hline \multirow{3}{*}{ Variável/ Categoria } & \multicolumn{5}{|c|}{ DIAGNÓSTICO } \\
\hline & anatomo desconhecido & beta persistente & mola completa & mola incompleta & neoplasia trofoblástica \\
\hline & $\mathbf{N}(\%)$ & $\mathbf{N}(\%)$ & $\mathrm{N}(\%)$ & $\mathbf{N}(\%)$ & $\mathbf{N}(\%)$ \\
\hline \multicolumn{6}{|l|}{ Faixa Etaria } \\
\hline$<20$ anos & $3(30,0)$ & $2(15,4)$ & $10(30,3)$ & $5(20,0)$ & $2(66,7)$ \\
\hline 20 a 34 anos & $7(70,0)$ & $10(76,9)$ & $14(42,4)$ & $16(64,0)$ & $1(33,3)$ \\
\hline$\geq 35$ anos & $0(0,0)$ & $1(7,7)$ & $9(27,3)$ & $4(16,0)$ & $0(0,0)$ \\
\hline \multicolumn{6}{|l|}{ ETNIA } \\
\hline Amarela & $1(10,0)$ & $1(10,0)$ & $1(3,7)$ & $0(0,0)$ & $0(0,0)$ \\
\hline Branca & $1(10,0)$ & $2(20,0)$ & $1(3,7)$ & $3(13,0)$ & $0(0,0)$ \\
\hline negra & $1(10,0)$ & $1(10,0)$ & $1(3,7)$ & $3(13,0)$ & $0(0,0)$ \\
\hline Parda & $7(70,0)$ & $6(60,0)$ & $24(88,9)$ & $17(73,9)$ & $2(100,0)$ \\
\hline \multicolumn{6}{|l|}{ COR/Raça } \\
\hline Branca & $2(20,0)$ & $3(30,0)$ & $2(7,4)$ & $3(13,0)$ & $0(0,0)$ \\
\hline Não Branca & $8(80,0)$ & $7(70,0)$ & $25(92,6)$ & $20(87,0)$ & $2(100,0)$ \\
\hline \multicolumn{6}{|l|}{ Religião } \\
\hline católica & $3(42,9)$ & $4(57,1)$ & $12(57,1)$ & $10(55,6)$ & $0(0,0)$ \\
\hline Outras religiões & $1(14,3)$ & $0(0,0)$ & $6(28,6)$ & $6(33,3)$ & $0(0,0)$ \\
\hline SEM religião & $3(42,9)$ & $3(42,9)$ & $3(14,3)$ & $2(11,1)$ & $2(100,0)$ \\
\hline \multicolumn{6}{|l|}{ Escolaridade } \\
\hline $1^{\circ}$ grau incompleto/ $1^{\circ}$ grau completo & $2(25,0)$ & $2(25,0)$ & $11(50,0)$ & $7(31,8)$ & $1(50,0)$ \\
\hline $2^{\circ}$ grau incompleto $/ 2^{\circ}$ grau completo & $4(50,0)$ & $6(75,0)$ & $9(40,9)$ & $12(54,5)$ & $1(50,0)$ \\
\hline 3 grau incompleto $/ 3^{\circ}$ grau completo & $2(25,0)$ & $0(0,0)$ & $2(9,1)$ & $3(13,6)$ & $0(0,0)$ \\
\hline \multicolumn{6}{|l|}{ SITUAÇÃOCONJUGAL } \\
\hline COM parceiro & $7(87,5)$ & $8(100,0)$ & $23(88,5)$ & $22(95,7)$ & $2(100,0)$ \\
\hline SEM parceiro & $1(12,5)$ & $0(0,0)$ & $3(11,5)$ & $1(4,3)$ & $0(0,0)$ \\
\hline \multicolumn{6}{|l|}{ Renda Familiar } \\
\hline$\leq 1$ salário mínimo & $4(57,1)$ & $4(57,1)$ & $17(85,0)$ & $13(72,2)$ & $1(100,0)$ \\
\hline 1 a 2 salários mínimos & $2(28,6)$ & $3(42,9)$ & $2(10,0)$ & $3(16,7)$ & $0(0,0)$ \\
\hline > 2 salários mínimos & $1(14,3)$ & $0(0,0)$ & $1(5,0)$ & $1(5,6)$ & $0(0,0)$ \\
\hline Sem renda fixa & $0(0,0)$ & $0(0,0)$ & $0(0,0)$ & $1(5,6)$ & $0(0,0)$ \\
\hline \multicolumn{6}{|l|}{ LUGAR } \\
\hline Capital & $3(27,3)$ & $2(16,6)$ & $9(30,0)$ & $6(24,0)$ & $0(0,0)$ \\
\hline Interior & $8(72,7)$ & $10(83,3)$ & $19(63,3)$ & $18(72,0)$ & $1(50,0)$ \\
\hline Outro estado & $0(0,0)$ & $0(0,0)$ & $2(6,7)$ & $1(4,0)$ & $1(50,0)$ \\
\hline \multicolumn{6}{|l|}{ Gestações } \\
\hline 1 gestação & $4(36,4)$ & $4(30,8)$ & $13(39,4)$ & $12(46,2)$ & $1(33,3)$ \\
\hline 2 ou mais gestações & $7(63,6)$ & $9(69,2)$ & $20(60,6)$ & $14(53,8)$ & $2(66,7)$ \\
\hline \multicolumn{6}{|l|}{ Paridade } \\
\hline Nenhuma & $4(36,4)$ & $5(38,5)$ & $13(39,4)$ & $14(53,8)$ & $1(33,3)$ \\
\hline 1 paridade & $3(27,3)$ & $5(38,5)$ & $7(21,2)$ & $9(34,6)$ & $2(66,7)$ \\
\hline mais de 1 & $4(36,4)$ & $3(23,1)$ & $13(39,4)$ & $3(11,5)$ & $0(0,0)$ \\
\hline
\end{tabular}

Fonte: Autores.

\section{Discussão}

A FIGO afirma que a DTG é mais prevalente nos extremos de idade reprodutiva, com aumento do risco especialmente após os 35 anos e há um risco 5 a 10 vezes maior após os 45 anos (Ngan, et al., 2018). No entanto, no presente estudo, e em consonância com estudo realizado na Bahia por Soares, et al. (2010), pudemos verificar a idade média de $26,7( \pm 8,7)$ anos e uma porcentagem de $42,9 \%$ com idade inferior a 35 anos no HU-UFS.

Foi possível observar também que a maioria das pacientes do serviço provém de famílias de baixa renda e baixo nível de escolaridade. Essa característica pode ser atribuída ao fato de que as pacientes encaminhadas ao Centro de Referência do HUUFS eram, em sua maioria, provenientes de maternidades públicas do estado, sendo, portanto, pouco referenciadas pacientes do sistema particular que possuem maior poder aquisitivo e maior escolaridade. Entretanto, estudos já realizados em outros continentes, e inclusive no Brasil, encontraram relação semelhante (Soares, et al.,2010). 
Research, Society and Development, v. 10, n. 3, e4110312934, 2021

(CC BY 4.0) | ISSN 2525-3409 | DOI: http://dx.doi.org/10.33448/rsd-v10i3.12934

A cor parda prevalente no estudo também foi evidenciada no estudo de Oliveira, et. al (2020) que apresentou uma porcentagem de $89,8 \%$. O que está relacionado também com a região em que os estudos foram feitos.

O serviço por ser referência no estado de Sergipe para DTG recebe muitas pacientes de outros municípios de Sergipe, no estudo estavam presentes pacientes de Lagarto, São Cristóvão, Nossa Senhora do Socorro, Barra dos Coqueiros, Itaporanga D’Ajuda, Arauá, Poço Verde, Monte Alegre, Indiaroba, Laranjeiras, Pirambu, Frei Paulo, Japoatã, Riachão do Dantas, Poço Redondo, Carmópolis, Simão Dias, Santana do São Francisco, Pedrinhas, Boquim, Propriá, Pedra Mole, Cristinápolis, Divina Pastora, Siriri, Itabaianinha e Itabaiana. Assim como pacientes provenientes de cidades de estados vizinhos como Penedo (AL), Jandaíra (BA), Fátima (BA) e Rio Real (BA).

A multiparadidade foi mais prevalente do que a nuliparidade no estudo, em consonância com Luarain (2010) que relaciona a paridade a mola e traz a multiparidade como maior fator de risco para o seu desenvolvimento.

No estudo, também foi possível observar a prevalência da Mola Completa $(38,4 \%)$ em relação a Mola Incompleta $(30,2 \%)$. O que também foi visto, mas com maior diferença na pesquisa de Pinto, et al. (2016), desenvolvida em Recife, que encontrou o percentual de $74 \%$ e $17,1 \%$ respectivamente e no estudo de Oliveira, et al. (2020) que foi de $43,8 \%$ e $31,3 \%$ respectivamente.

Em relação a um estudo semelhante desenvolvido em outra capital do nordeste, a forma de esvaziamento uterino foi de 44,4\% por AMIU e 37,9\% por curetagens, como métodos isolados (Oliveira, et al., 2020). Enquanto em Aracaju ocorrem 51,9\% de curetagens e 30,4\% por AMIU. Evidenciando em Aracaju a prevalência maior de curetagens em relação à outra capital nordestina, o que pode estar associado a complicações, como perfuração uterina e formação de sinéquias (Moraes et al., 2014).

Quanto a evolução para Neoplasia Trofoblástica Gestacional, termo utilizado para as formas malignas da doença, um dos maiores estudos do Brasil trás que 24,6\% das MHC e 7,6\% das MHP progrediram para esta forma (Braga, et al., 2014). No estudo aqui apresentado pudemos observar que 12,8\% tiveram evolução do diagnóstico para malignidade.

\section{Conclusão}

O perfil predominante encontrado no estudo é de mulheres com idade acima de 35 anos, pardas, com escolaridade até o $2^{\circ}$ grau completo, com história prévia de gestação, com parceria presente, que possuem renda mensal até 1 salário-mínimo e vêm do interior de Sergipe para a capital para receber tratamento no Centro de Referência do Estado para DTG.

Como limitações deste estudo encontram-se o reduzido número de pacientes e perda de seguimento de alguns pacientes ainda no primeiro ano após o diagnóstico de Doença Trofoblástica Gestacional. Para trabalhos futuros, sugerem-se a avaliação de um maior número de pacientes e avaliação imuno-histoquímica para diferenciação das neoplasias trofoblásticas.

\section{Referências}

Braga, A. (2018). Doença trofoblástica gestacional. Federação Brasileira das Associações de Ginecologia e Obstetrícia (Febrasgo). (Protocolo Febrasgo Obstetrícia, no 23/Comissão Nacional Especializada em Doença Trofoblástica Gestacional). 47(1): 6-17.

Braga, A., Lin, L. H., Maestá, I., Sun, S., Yazaki, U., Elza, M., José, M., \& Viggiano, M. (2019). Gestational Trophoblastic Disease in Brazil. Revista Brasileira de Ginecologia e Obstetrícia, 41(4), 211-212

Braga, A., Uberti, E., M. H., Fajardo, M. C., Viggiano, M., Sun, S. Y., Grillo, B. M. et al. (2014) Epidemiological report on the treatment of patients with gestational trophoblastic disease in 10 Brazilian referral centers. Results after 12 years since International FIGO 2000 consensus. J Reprod Med. 59(5-6): 2417 .

FIGO Oncology Committee. (2002). FIGO staging for gestational trophoblastic neoplasia 2000. FIGO Oncology Committee. Int J Gynaecol Obstet. 77(3):2857.

Genest, D. R., Berkowitz, R. S., \& Fisher, R. A. Gestational trophoblastic disease. In: Tavassoli, F. A., Devilee, P., editores. World Health Organization classification of tumours. Pathology and genetics: tumors of the breast and female genital organs: IARC Press; 2003. $250-6$. 
Research, Society and Development, v. 10, n. 3, e4110312934, 2021

(CC BY 4.0) | ISSN 2525-3409 | DOI: http://dx.doi.org/10.33448/rsd-v10i3.12934

Gockley, A. A., Melamed, A., Joseph, N. T., Clapp, M., Sun, S. Y., Goldstein, D. P., Horowitz, N. S., \& Berkowitz, R. S. (2016). The effect of adolescence and advanced maternal age on the incidence of complete and partial molar pregnancy. Gynecologic oncology, 140(3), 470-473.

Ireson, J., Jones, G., Winter, M. C., Radley, S. C., Hancock, B. W., \& Tidy, J. A. (2018). Systematic review of health-related quality of life and patient-reported outcome measures in gestational trophoblastic disease: a parallel synthesis approach. The Lancet. Oncology, 19(1), e56-e64.

Khoo, S. K. (2003). Clinical aspects of gestational trophoblastic disease: a review based partly on 25 -year experience of a statewide registry. The Australian \& New Zealand journal of obstetrics \& gynaecology, 43(4), 280-289.

Lurain, J. R. (2010). Gestational trophoblastic disease I: epidemiology, pathology, clinical presentation and diagnosis of gestational trophoblastic disease, and management of hydatidiform mole. American journal of obstetrics and gynecology, 203(6), 531-539.

Melamed, A., Gockley, A. A., Joseph, N. T., Sun, S. Y., Clapp, M. A., Goldstein, D. P., Berkowitz, R. S., \& Horowitz, N. S. (2016). Effect of race/ethnicity on risk of complete and partial molar pregnancy after adjustment for age. Gynecologic oncology, 143(1), 73-76.

Moraes, V. P. D., Marcolino, L. A., Sá, R. A. M. D., Silva, E. P. D., Amim Júnior, J., Rezende Filho, J. F. D., \& Braga, A. (2014). Complicações clínicas da gravidez molar. Femina, 42(5), 229-234.

Ngan, H., Seckl, M. J., Berkowitz, R. S., Xiang, Y., Golfier, F., Sekharan, P. K., Lurain, J. R., \& Massuger, L. (2018). Update on the diagnosis and management of gestational trophoblastic disease. International journal of gynaecology and obstetrics: the official organ of the International Federation of Gynaecology and Obstetrics, 143 Suppl 2, 79-85.

Pereira, A. S. et al. (2018). Metodologia da pesquisa científica. UFSM. https://repositorio.ufsm.br/bitstream/handle/1/15824/Lic_Computacao_MetodologiaPesquisa-Cientifica.pdf?sequence $=1 \mathrm{em} 24$ de fevereiro de 2021.

Pinto, L. T. C., Costa, A. A. R., Lira, C. M. P., Melo, L. B., \& Borba, S. K. M. (2016). Frequência, características clínicas e evolução de pacientes com doença trofoblástica gestacional que desenvolveram complicações. Instituto de Medicina Integral Prof. Fernando Figueira - IMIP. Programa de Iniciação Científica.

Oliveira H. G. S., Santos A. A. P., Lucena T. S., Sanches M. E. T. L., Vieira M. J. O., Teixeira L. M., Oliveira J. C. S., \& Rodrigues R. P. G. T. O. (2020), Panorama da doença trofoblástica gestacional numa capital do nordeste. Revista Eletrônica Acervo Saúde, 12(9), e4212.

Soares, P. D., Maestá, I., Costa, O. L., Charry, R. C., Dias, A., \& Rudge, M. V. (2010). Geographical distribution and demographic characteristics of gestational trophoblastic disease. The Journal of reproductive medicine, 55(7-8), 305-310.

Sun, R., Zhang, Y., Zheng, W., Tian, Q., An, R., \& Xue, Y. (2016). Clinical Characteristics of Gestational Trophoblastic Neoplasia: A 15-Year Hospital-Based Study. International journal of gynecological cancer: official journal of the International Gynecological Cancer Society, 26(1), 216-221.

Zugaib, M. (2020). Zugaib Obstetrícia: Malone. (4th ed.,) 611-626. 